\title{
Nonlinear electrical transport in K-TCNQ at low temperatures
}

\author{
D P GOSWAMI and M L KALRA \\ Department of Physics, M. L. Sukhadia University, Udaipur 313001, India \\ MS received 20 May 1993; revised 4 August 1993
}

\begin{abstract}
A remarkable nonlinearity has been observed for electric transport in K-TCNQ (potassium tetracyanoquinodimethane) quasi-one-dimensional conductor. The negative differential resistance region appears after $V_{m}$ (turn over voltage), beyond which voltage goes down with increase of current. A possible mechanism is discussed in terms of dynamics of charged solitons and domain walls in one-dimensional molecular stacks of these types of crystals.
\end{abstract}

Keywords. One-dimensional conductors; nonlinear conduction; negative differential resistance; solitons; radical ion salts.

\section{Introduction}

The interest in one-dimensional conductors arose out of the work of Little (1964), who proposed that in certain organometallic structures high temperature superconductivity should in principle be possible. Radical ion salts grown with TCNQ (tetracyanoquinodimethane) as an acceptor are found to have probably the least resistivity at room temperature (Melby et al 1962). K-TCNQ (potassium-TCNQ) single crystal synthesized by us has a room temperature resistivity of about $4977.6 \Omega \mathrm{cm}$ and is found to exhibit semiconducting behaviour in the temperature range studied ( $70 \mathrm{~K}$ $300 \mathrm{~K}$ ). The value of $\sigma_{\|} \mid \sigma_{\perp}$ is found to be of the order of 40 at room temperature and hysteresis is not observed. Below $100 \mathrm{~K}$ the resistivity of the single crystal was found to be almost constant. The $I-V$ characteristic of K-TCNQ has been studied at $221 \mathrm{~K}$ which shows a marked deviation from ohmicity. The non-ohmic behaviour is explained on the basis of dynamics of charged solitons and domain walls in the one-dimensional molecular stacks of these types of crystals. The same argument has been applied for the discussion of linear and nonlinear conductivities in mixed stácks of CT crystals (Nagaosa 1986) and for the negative resistance region in Rb-TCNQ (Watanabe 1991).

\section{Experimental}

K-TCNQ has been synthesized by the method due to Melby et al (1962) with acetonitrile as the solvent medium. It has been characterized by the usual spectroscopic, $\mathrm{X}$-ray diffraction and microanalysis techniques.

Elemental analysis was done to compare observed percentage composition of the constituent elements to the calculated one. Table 1 shows the observed percentage composition of the complex grown along with the calculated one.

Infrared absorption spectra can be taken using nujol mulls or pellets with $\mathrm{KBr}$ in the IR range $\left(400-4000 \mathrm{~cm}^{-1}\right)$ to determine whether the complex formed is ionic or 
Table 1. Percentage composition of K-TCNQ.

\begin{tabular}{lccccccccc}
\hline & \multicolumn{3}{c}{ Calculated value } & \multicolumn{4}{c}{ Observed value } \\
\cline { 2 - 5 } & $\mathrm{C}$ & $\mathrm{H}$ & $\mathrm{N}$ & Metal & $\mathrm{C}$ & H & N & Metal \\
\hline K-TCNQ & 59.25 & 1.6 & 23.04 & 16.04 & 59.5 & 1.54 & 23.79 & 16.33 \\
\hline
\end{tabular}

Table 2. Main infrared peaks of organic radical ion salt in $\mathrm{cm}^{-1}$.

\begin{tabular}{lccc}
\hline Complex & $\mathrm{C} \equiv \mathrm{N}$ & $\mathrm{C}=\mathrm{C}$ & Quinoid skeletal bands \\
\hline TCNQ & $2220(\mathrm{~s}, \mathrm{sp})$ & $1540(\mathrm{~s}, \mathrm{sp})$ & $1350(\mathrm{~s}, \mathrm{sp})$ \\
& & $1130(\mathrm{~m}, \mathrm{sp})$ \\
& & $960(\mathrm{~m}, \mathrm{sp})$ \\
& & $860(\mathrm{vs}, \mathrm{sp})$ \\
K-TCNQ & $2180(\mathrm{~ms}, \mathrm{sp})$ & $1508(\mathrm{~m}, \mathrm{sp})$ & $1350(\mathrm{~s}, \mathrm{sp})$ \\
& & & $1160(\mathrm{~m}, \mathrm{br})$ \\
& & $950(\mathrm{~m}, \mathrm{br})$ \\
& & $720(\mathrm{~m}, \mathrm{sp})$ \\
\hline
\end{tabular}

not. In table 2 are shown the main IR peaks of the radical ion salt K-TCNQ and TCNQ.

Visible absorption measurements give information about the composition of the complex. Spectra are taken either in solid or in appropriate solution in which it dissociates into compounds of their neutral form. The absorption spectrum of $\mathrm{K}$ TCNQ was taken in acetone. It consists of peaks at $850,750,680$ and $395 \mathrm{~nm}$. For complex salt in solution, components are found to be completely dissociated giving spectrum consisting of independent TCNQ and $\mathrm{TCNQ}^{-}$molecules. The bands at $680 \mathrm{~nm}$ and $395 \mathrm{~nm}$ nearly correspond to $641 \mathrm{~nm}$ and $370 \mathrm{~nm}$ respectively in the dimer. Band at $750 \mathrm{~nm}$ not only corresponds to those of dimer but also that due to neutral TCNQ. Band at $850 \mathrm{~nm}$ may be charge-transfer band between $\mathrm{TCNQ}^{-}$molecules.

Crystals of K-TCNQ have been analysed by X-ray diffraction by Konno et al (1977). The single crystal data show that the crystal structure is monoclinic in nature with cell parameters $a=7.0835 \AA, b=17.733 \AA, c=17.859 \AA, \beta=94.95$ and $z=8$ and the space group is $p_{1}^{2} / n$. Here $a$ is the crystal axis, and $b$ and $c$ are the two axes defining the plane containing the TCNQ molecules.

All low temperature measurements were carried out using two-probe method in a closed cycle liquid He cryostat because of the very small size of the crystals. Electrical connections were provided to the sample using silver paste and the current source was used as a driving source. The voltage across the two points on the sample was monitored as a function of current.

\section{Results}

Figure 1 shows the $I-V$ characteristic at $221 \mathrm{~K}$. As is evident from the figure, the behaviour of the system is nonlinear. At very low currents the characteristic is almost 
linear or ohmic. Pronounced nonlinearity is observed beyond a current of $300 \mathrm{nA}$. The negative differential resistance region appears after the $V_{m}$ (turn over voltage) of $50 \mathrm{mV}$ at $800 \mathrm{nA}$ current beyond which voltage goes down with increase of current. It is found that $\sigma_{\text {less resistive }} / \sigma_{\text {high resistive }} \approx 1.2$ in the threshold region. Region I (ohmic) has high resistivity and region II (non-ohmic) has comparatively less resistivity.

\section{Discussion}

A deviation from ohmic behaviour at high driving field has been reported in a number of compounds such as TTF-TCNQ (Gunning and Hegger 1978), Qn(TCNQ) ${ }_{2}$ pellet (Goswami et al 1992), $\mathrm{NbSe}_{3}$ and $\mathrm{TaS}_{3}$ (Fleming and Grimes 1979). The $I-V$ characteristic of our system shows that at some current $I_{m}$ voltage attains a maximum value $V_{m}$ known as the turn over voltage. Beyond this point as the current increases the voltage decreases and the system shows a negative differential resistance. The values of $V_{m}$ and $I_{m}$ for our system are $50 \mathrm{mV}$ and $800 \mathrm{nA}$ respectively. The overall behaviour can be explained qualitatively as follows.

Recently, Okamoto et al (1984) measured IR molecular-vibration spectra of K-TCNQ and Rb-TCNQ crystals and found that the dimeric lattice distortion in those crystals near $T_{c}=395 \mathrm{~K}$ fluctuates both temporally and spatially. It has also been proposed that such a structural fluctuation in the dimerized lattice is due to the thermal excitation of mobile kink-soliton-type defects in the dimerized TCNQ columns, as schematically illustrated in figure 2 for \pm charged solitons. This figure illustrates the connection between charged solitons and radical ion salts. In this sense, they are regarded as the counterparts of the well-known charged kink solitons in transpolyacetylene having a degenerate bond-ordered-wave (BOW) ground state (Okamoto et al 1984).

When an electric field is applied to the crystal, the charge defects in the form of solitons are driven into motion. At low fields, however, the free motion of individual carriers (charged solitons) is hindered by the interstack interaction. The linear voltage at low field may be attributed to the motion of a few defects which are more or less free from the interstack interaction, while the majority of defects are locked in the

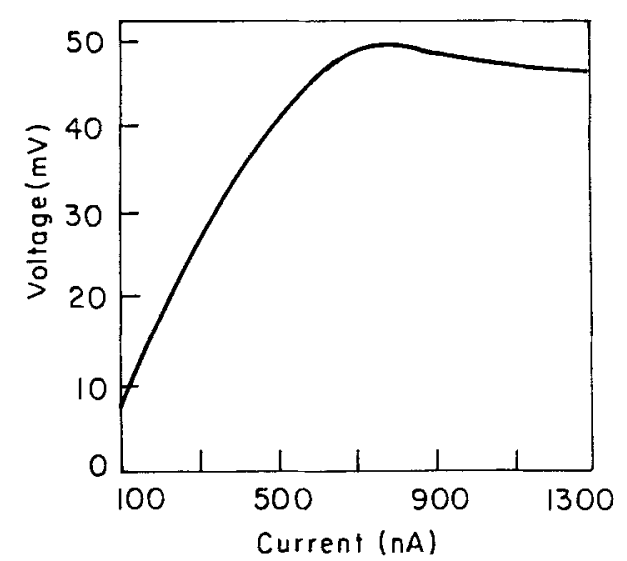

Figure 1. Current-voltage characteristics of $\mathrm{K}$ TCNQ single crystal at $221 \mathrm{~K}$.

\section{Charged solitons}

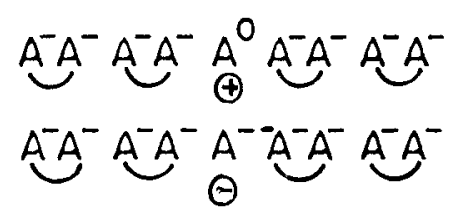

Figure 2. Schematic structures of the acceptor (A) stack: charged solitons. 
three-dimensional order. When the electric field is increased further to a certain threshold value $E_{T}$ (here $E_{T}=321.4 \mathrm{~V} / \mathrm{cm}$ ), a sudden break of three-dimensional order in the BOW state takes place. Once this occurs individual charged defects can now move frequently. The sudden change is brought about by a positive feedback process in which the field-induced motion of defects leads to an accelerated loosening of the interstack interaction. Due to this, the voltage drops as the current increases and thus negative differential resistance region appears. Thus we can think of the negative resistance as 'a current-induced transition' from the bulk BOW state to the state of the reduced order (Watanabe 1991).

\section{Conclusion}

Detailed experiment on the $I-V$ characteristics of an organic quasi-one-dimensional conductor K-TCNQ at a particular low temperature has clearly demonstrated pronounced nonlinear conduction and negative differential resistance. These are explained on the basis of dynamics of charged solitons and domain walls in the one-dimensional molecular stacks of these types of crystals.

\section{Acknowledgements}

The authors are highly thankful to Prof. U N Upadhyaya for his support in pursuit of this work. Financial assistance from the Department of Science and Technology, New Delhi, is gratefully acknowledged.

\section{References}

Fleming R M and Grimes C C 1979 Phys. Rev. Lett. 421423

Goswami D P, Singh Y, Bala M and Kalra M L 1992 Pramana-J. Phys. 39351

Gunning W J and Hegger A J 1978 Solid State Commun. 27843

Konno M, Ihiha T and Saito Y 1977 Acta Crystallogr. B33 763

Little W A 1964 Phys. Rev. A134 1416

Melby L R, Harder R J, Herter W R, Mahler W and Benson R E 1962 J. Am. Chem. Soc. 843374

Nagaosa N 1986 J. Phys. Soc. Jpn 552754

Okamoto H, Tokura Y and Koda T 1984 Phys. Rev. Lett. 53842

Watanabe N 1991 Phys. Rev. B20 11111 